\title{
In Situ Observations of Carbon Nanotube Formation and Growth Process
}

\author{
Renu Sharma
}

Center for Solid State Science, Arizona State University, Tempe, Arizona 85287-1704.

Carbon nanotubes (CNTs) were dramatically novel nanoscale materials when they were first discovered in 1991 by Iijima using a carbon arc discharge process. ${ }^{1}$ A number of CNTs, differing both in structure (arm-chair, zig-zag and chiral) and layer thickness (e.g. multiwall, double wall and single wall) have been identified. They, specially single wall carbon nanotubes (SWCNT), have since become one of the most sought out materials for nanotechnology due to their remarkable magnetic, electronic and mechanical properties. Recently, catalytic chemical vapor deposition $(\mathrm{CVD})^{2,3}$ has been found to provide a more controlled, near-equilibrium route to both multi wall and single wall carbon nanotubes (MWCNT and SWCNT respectively) structures. Unfortunately, synthesis of a single phase of any one type of CNT is still difficult. For the first time, a modified Phillips 430 environmental transmission electron (ETEM) ${ }^{4}$, operated at $200 \mathrm{KV}$ is employed for insitu observations of CVD reaction conditions and growth mechanisms of carbon nanotubes (CNT).

Powder $\mathrm{Ni} / \mathrm{SiO}_{2}$ catalyst containing nanosized metal particles, was dry loaded on $\mathrm{Ni}$ grids and was heated up to $400^{\circ} \mathrm{C}$ either in vacuum or in one Torr of $\mathrm{H}_{2}$ gas inside the ETEM column, to ensure the $\mathrm{Ni}$ catalyst to be in pure metallic state (oxide free). The temperature was then raised to $500-700{ }^{\circ} \mathrm{C}$ and $\mathrm{H}_{2}$ was slowly replaced by $300-1000 \mathrm{~m}$ Torr of either propylene or acetylene gas (precursors for growing CNTs). Low magnification images were recorded using a TV rate camera located at the end of a Gatan imaging filter (GIF) connected to digital video recorder. High-resolution transmission electron microscopy (HREM) images were recorded, ex situ, using a JEOL 4000EX TEM.

Filamentous growth was observed when propylene was used as a precursor but these filaments were found to be pure graphitic in nature. Acetylene $\left(\mathrm{C}_{2} \mathrm{H}_{2}\right)$ gas precursor, on the other hand, resulted in the growth of well-defined nanotube structures. Figure 1 shows a time resolved series of images digitized from a video sequence recorded at $500^{\circ} \mathrm{C}$. Small metal particles, with dark contrast (Figure 1a), were observed to move ahead of a tubular structure (Figure 1b) and as the metal particle moved (left to right in the images) in a wavy manner, sometimes making a $360^{\circ}$ turn (Figure $1 \mathrm{c}$ ), the tubular structure was observed to grow. These tubular structures formed at low temperatures could be identified as MWCNT by HREM (Figure 2). The wavy nature of some of the tubes can also be observed in the HREM images. Sometimes the catalyst particle was observed to change its shape from round to elliptical in order to fit inside the tube diameter (Figure 2). As the reaction temperature was increased, the number of graphene walls in a nanotube decreased and above $700^{\circ} \mathrm{C}$ only SWCNT were observed to form (Figure 3). Ex-situ HREM observations after reaction confirmed the formation of CNTs all over the sample, thus ruling out any effect of electron beam on the growth process.

Thus the in-situ observations provide information about the reaction conditions for selective growth of SWNT as well as the growth mechanism. The measured growth rates also provide an insight on the activation energies for the formation of different type of CNTs. 

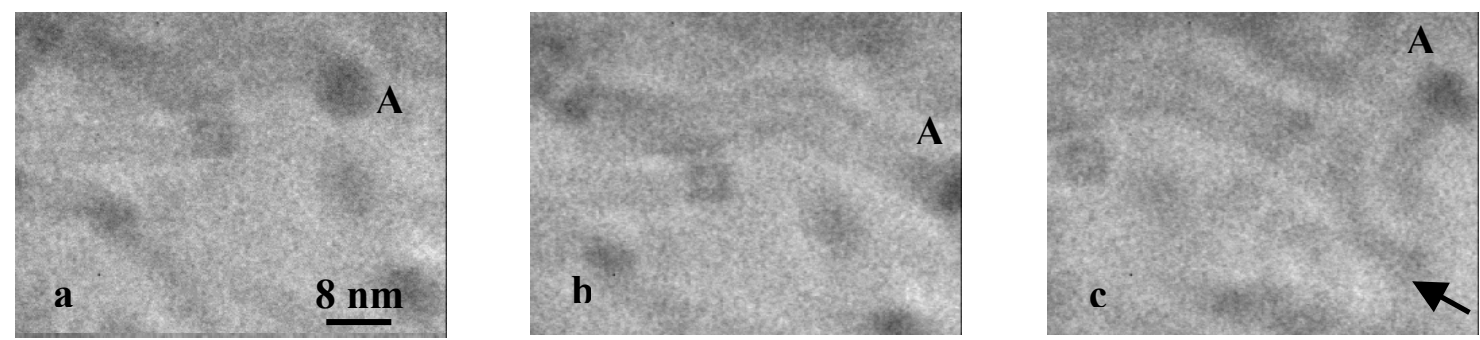

Figure 1 Low magnification images digitized from a video sequence showing the formation (a) and growth of CNT (b) as a catalyst particle (marked A) is observed to move from left to right in a wavy manner making a $360^{\circ}$ loop (c; marked by arrow). Note the formation and growth of other CNTs at the same time.

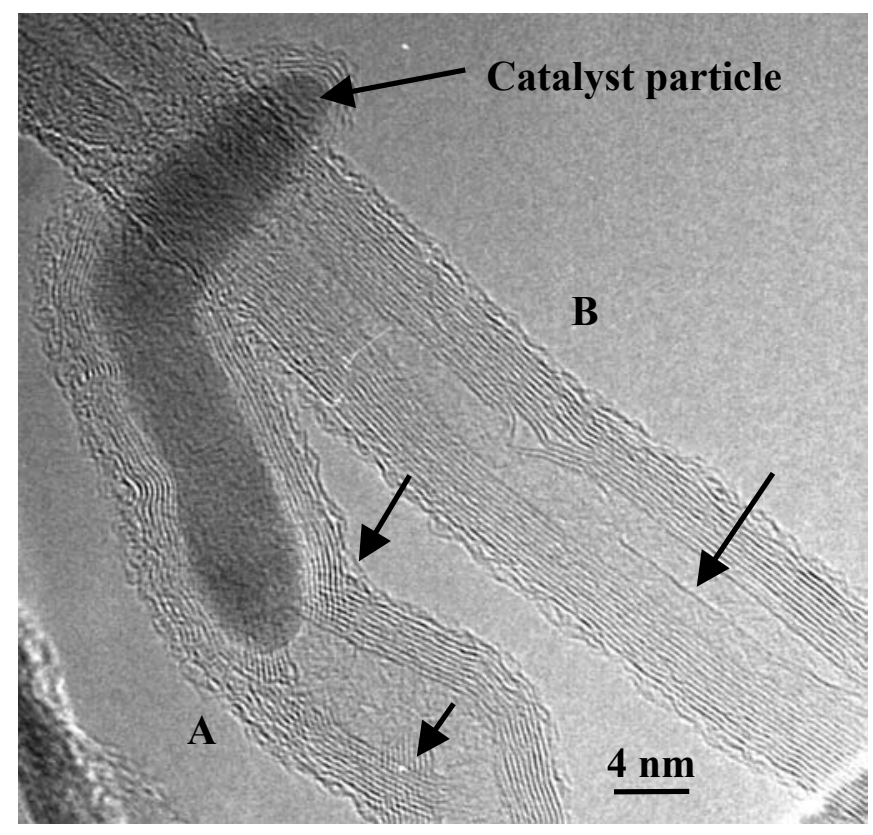

Figure 2. HREM image showing the wavy nature of a MWCNT (marked $A$ ) as observed during the growth at $500^{\circ} \mathrm{C}$ in acetylene and an elongated instead of a round catalyst particle is present at the apex of the CNT. Note the beginning and the end a new set of graphene layers at the curves of the CNT (marked by arrows). The second $M W C N T(B)$ shows the detached single graphene sheet (marked by arrow) present in the middle of the tube.

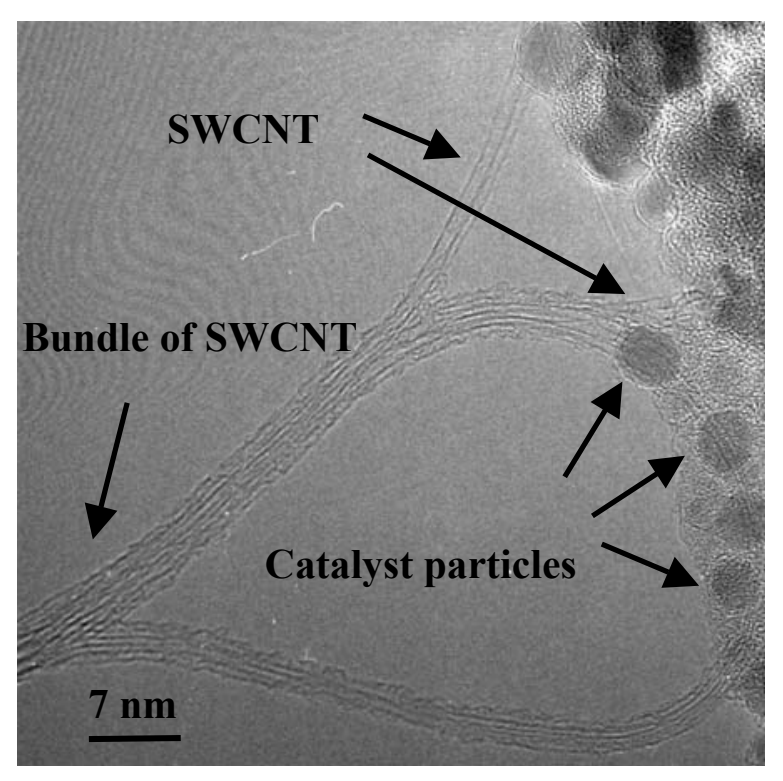

Figure 3. HREM image of SWCNT grown in the ETEM at $740^{\circ} \mathrm{C}$. The SWNT were often observed to grow from the catalyst sample without encapsulating the catalyst particle either at the base or at the apex and often joined together to form large bundles.

\section{Reference:}

1. S. Iijima, Nature, 354 (1992) 56.

2. M.J. Bronikowskiet al, J. Vac. Sci. Technol. A19, (2001) 1800.

3. Zhang et al, Appl. Phys. A 74 (2002) 325.

4. Sharma, R. and Weiss, K., Microscopy Research and Techniques, 42 ((1998) 270. 